Dom. Cien., ISSN: 2477-8818

Vol. 5, núm.1., ene, 2019, pp. 695-712

\title{
La auditoría forense aplicada a la investigación penal
}

\section{The forensic audit applied to criminal investigation}

\section{A auditoria forense aplicada à investigação criminal}

\author{
Dewis E. Álvarez-Pincay ${ }^{\mathrm{I}}$ \\ dewis.alvarez@unesum.edu.ec \\ Sandra P. Tóala-Bozada II \\ sandra.toala@unesum.edu.ec \\ Zoila M. Delgado-Gutierrez ${ }^{\text {III }}$ \\ zoila.delgado@unesum.edu.ec \\ José F. Peñafiel-Loor IV \\ jose.penafiel@unesum.edu.ec \\ Adela del Jesús Lucio-Pillasagua ${ }^{\mathrm{V}}$ \\ adela.pillasagua@unesum.edu.ec \\ Mercy K. Moreira-Cañarte VI \\ mercy.moreira@unesum.edu.ec
}

Recibido: 27 de noviembre de 2018 * Corregido: 18 de diciembre de 2018 * Aceptado: 20 de diciembre de 2018

\footnotetext{
I. Master en Dirección Estratégica; Licenciado en Contaduría y Auditoría; Docente Titular Principal de la Universidad Estatal del Sur de Manabí; Jipijapa, Ecuador.

II. PhD. en Administración; Magister en Docencia Universitaria e Investigación Educativa; Ingeniera Comercial; Docente de la Universidad Estatal del Sur de Manabí; Jipijapa, Ecuador.

III. Maestría en Contabilidad y Auditoría; Economista; Docente Contratada de la Universidad Estatal del Sur de Manabí; Jipijapa, Ecuador.

IV. Magister en Gerencia Educativa; Ingeniero Comercial; Docente Titular Principal de la Universidad Estatal del Sur de Manabí; Jipijapa, Ecuador.

v. Magister en Gerencia Educativa; Ingeniera en Contabilidad y Auditoría; Docente Contratada de la Universidad Estatal del Sur de Manabí; Jipijapa, Ecuador.

VI. Magister en Tributación y Finanzas; Ingeniera Comercial; Docente Contratada de la Universidad Estatal del Sur de Manabí; Jipijapa, Ecuador.
} 


\title{
Resumen
}

El Delito o Crimen es tan antiguo como la sociedad, los hombres que tenían en sus manos el poder de administrar justicia han necesitado demostrar la inocencia o culpabilidad de aquellos que infringían las normas, ya sea para castigar al infractor o para absolver al inocente. De allí, que la búsqueda de la verdad a través de los medios de prueba, ha ido evolucionando desde las antiguas “ordalías” en las que el Juez supremo decidía la inocencia o culpabilidad de una persona. Actualmente y de manera unánime, la doctrina señala que para lograr la consolidación del Moderno Proceso Penal y los diferentes sistemas que lo operativizan, es necesario apoyarse en las tecnologías de vanguardia que ofrecen las ciencias forenses, las cuales representan una herramienta para la lucha contra el fraude y la corrupción, su actuación facilita las evidencias necesarias para enfrentar los delitos en el sector público y privado por medio de investigaciones donde se emiten opiniones de valor técnico que le permiten a la justicia actuar con mayor certeza. Su implementación se origina, cuando las auditorias tradicionales no son suficientes y nace esta con los intentos por detectar e investigar el fraude desarrollando técnicas específicas que permiten combatir el crimen y alcanzar la justicia. En función de esto, se establece la importancia del presente artículo, por cuanto se encuentra centrado en analizar la auditoría forense entendida como un Instrumento Jurídico Contable, diseñado para la obtención de pruebas de carácter público y privado, aplicable a los delitos de fraudes contables en un litigio o proceso judicial; permitiendo de este modo que esté al alcance de la sociedad, sea transparente y profesional y que brinde la posibilidad de otorgarle celeridad y relevancia a los procesos jurídicos en esta materia.

Palabras claves: Auditoría Forense; Investigación Penal; Fraudes; Crímenes Contables; Justicia.

\begin{abstract}
The crime or the crime is as old as the society, the man who had the power to administer justice, the duty to prove the innocence or the guilt of the offenders of the rules, the sea for error and error. From there, that the search for truth through the means of proof, has evolved from the ancient "ordeals" in which the supreme judge decided the innocence or guilt of a person. Currently and in a united way, the doctrine states that to achieve the consolidation of the Modern Criminal Process and the different systems that operate, it is necessary to rely on the avant-garde technologies offered by the forensic sciences, which represents a tool for the fight against corruption. In this case, opinions
\end{abstract}

\footnotetext{
696 Vol. 5, núm. 1, enero 2019, pp. 695-712

Dewis E. Álvarez-Pincay; Sandra P. Tóala-Bozada; Zoila M. Delgado-Gutierrez; José F. Peñafiel-

Loor; Adela del Jesús Lucio-Pillasagua; Mercy K. Moreira-Cañarte
} 
of technical value that allow you to act correctly are published. Its implementation originates when traditional audits are not enough and are not more frequent. Based on this, the importance of this article is shown, which is why it is focused on the analysis of the Understanding function as a Legal Accounting Instrument, designed to obtain public and private evidence, applicable to fraud crimes accountants in a litigation or judicial process; This is the reason why it will be granted to society and the importance of legal processes in this matter.

Key words: Forensic audit; Criminal Investigation; Frauds; Accounting Crimes; Justice.

\section{Resumo}

O crime ou o crime é tão antigo quanto a sociedade, o homem que tinha o poder de administrar a justiça, o dever de provar a inocência ou a culpa dos infratores das regras, o mar por erro e erro. A partir daí, que a busca da verdade através dos meios de prova, evoluiu a partir das antigas "provações" em que o juiz supremo decidiu a inocência ou culpa de uma pessoa. Atualmente e de forma unida, a doutrina afirma que, para alcançar a consolidação do Processo Penal Moderno e dos diferentes sistemas que operam, é necessário contar com as tecnologias de vanguarda oferecidas pelas ciências forenses, o que representa uma ferramenta para o luta contra a corrupção. Nesse caso, as opiniões de valor técnico que permitem que você aja corretamente são publicadas. Sua implementação se origina quando as auditorias tradicionais não são suficientes e não são mais frequentes. Com base nisso, a importância deste artigo é mostrada, razão pela qual se concentra na análise da função Understanding como um Instrumento de Contabilidade Legal, projetado para obter evidência pública e privada, aplicável a contadores de fraudes em um processo contencioso ou judicial. ; Essa é a razão pela qual ela será concedida à sociedade e à importância dos processos legais nessa questão.

Palavras chave: Auditoria Forense; Investigação Criminal; Fraudes; Crimes Contábeis; Justiça. 


\section{Introducción.}

Actualmente, los delitos económicos representan una de las principales preocupaciones de las organizaciones, estos reflejan a algunos individuos de una sociedad deseosa de obtener un estatus económico de una manera fácil sin importar valores éticos y morales; los porcentajes de pérdidas que las entidades tienen por esta causa, son un ejemplo representativo para combatirla; es así que las empresas y los organismos legales, en su afán de lucha han desarrollado mecanismos para detectar y controlar estos ilícitos como normativas, programas o herramientas de control y todo tipo de actividades especializadas en la prevención y detección del fraude, para así tratar de evitar y minimizar el decrecimiento de la economía.

En este contexto y dada la importancia que han adquirido estos ilícitos, se ha visto la necesidad de buscar alternativas como la Auditoria Forense, una herramienta para la lucha contra el fraude y la corrupción, que se ha convertido en una opción para combatir el fraude, facilitando las evidencias necesarias para enfrentar los delitos en el sector público y privado por medio de investigaciones donde se emiten opiniones de valor técnico que le permiten a la justicia actuar con mayor certeza.

Según Venziatt (2014).

El fraude, es conceptualizado como aquel hecho que se caracteriza por acciones que se enmarcan dentro del contexto de realizar intencionalmente una representación falsa sobre una acción o acontecimiento importante. Dicha representación se ampara en normas lícitas de hecho y derecho o en procedimientos lícitos establecidos, la cual causará a la víctima pérdidas de dinero y/o propiedades. (p.65).

Lo referido lleva a interpretar que el fraude se asocia con una acción apoyada en el engaño intencional, este hecho puede ser cometido en oficinas públicas y privadas, compañías de seguros, instituciones financieras, bolsas de valores, industria petrolera, constructoras y en general, en toda entidad en donde exista la posibilidad de cometer un engaño camuflado bajo la apariencia de legalidad.

Dicho de esta forma, se entiende que la existencia y comisión de fraudes y delitos como tipos delictivos, son la base para la creación de un modelo de Auditoría Forense, definida por Barrett

\footnotetext{
698 Vol. 5, núm. 1, enero 2019, pp. 695-712

Dewis E. Álvarez-Pincay; Sandra P. Tóala-Bozada; Zoila M. Delgado-Gutierrez; José F. Peñafiel-

Loor; Adela del Jesús Lucio-Pillasagua; Mercy K. Moreira-Cañarte
} 
(2015), como "el estudio dirigido a un fraude administrativo o contables, el cual representa uno de los que más auge ha tomado en los últimos tiempos". (p.23). En este sentido, se considera a la Auditoría como el proceso a través del cual se acumulan y evalúan las evidencia acerca de la información cuantitativa de una entidad económica específica, con el propósito de determinar e informar sobre el grado de correspondencia existente entre la información cuantificable y los criterios establecidos.

Dentro de estas características y considerando la evolución de la sociedad y de sus organizaciones, así como los avances tecnológicos y la diversidad de delitos que la afectan, a finales del siglo XX se empieza a hablar de la Auditoría Forense, como producto de su reaparición en los Estados Unidos, como una herramienta para suministrar prueba a los fiscales. Al hablar de Auditoría Forense no se habla sólo de una técnica nueva ni del nacimiento de una nueva ciencia, sino de la conjunción de varias ciencias encabezadas por la Contaduría Pública y el Derecho.

De esta manera, se comienza a aplicar la idea de una auditoría especializada basada en la contabilidad forense. Este tipo de auditoría nace cuando se vincula lo legal con los registros y pruebas contables. Así pues, la Contaduría se ha especializado en descubrir, evaluar y divulgar el flagelo de la corrupción, a fin de lograr el cumplimiento del desarrollo de las funciones públicas y privadas, conforme a normas y reglas.

En esta línea de ideas, Kartt (2015), establece que desde el punto de vista contable:

La auditoría forense es considerada como un examen sistemático de los estados financieros, contables, administrativos, operativos y de cualquier otra naturaleza, el cual permite determinar el cumplimiento de principios económico-financieros, la adherencia a los principios de contabilidad generalmente aceptados, el proceso administrativo y las políticas de dirección, normas y otros requerimientos establecidos por la organización. (p.12)

Desde esta perspectiva, se puede decir que la Auditoría es la actividad por la cual se verifica la corrección contable de las cifras de los estados financieros, se basa en la revisión de los registros, cálculos y fuentes de contabilidad para determinar la racionabilidad de las cifras que muestran los estados financieros y se puede clasificar principalmente en dos grandes grupos, uno externo y otro

\footnotetext{
699 Vol. 5, núm. 1, enero 2019, pp. 695-712

Dewis E. Álvarez-Pincay; Sandra P. Tóala-Bozada; Zoila M. Delgado-Gutierrez; José F. PeñafielLoor; Adela del Jesús Lucio-Pillasagua; Mercy K. Moreira-Cañarte
} 
interno. Este tipo de Auditoría se lleva a cabo cuando se tiene la intención de publicar el producto del sistema de información examinado, con el fin de acompañar al mismo con una opinión independiente que le dé autenticidad y permita a los usuarios de dicha información tomar decisiones, confiando en las declaraciones del auditor.

En unión de estos planteamientos Gumter y Liiutt (2014), sostienen que existen dos tipos de auditorías forenses, la externa y la interna: "la auditoría externa debe hacerla una persona o firma independiente de capacidad profesional reconocida, capaz de ofrecer una opinión imparcial y profesionalmente experta acerca de los resultados de auditoría”. (p.32). En tal caso, se entiende que la auditoría externa debe estar basada en el hecho de que su opinión ha de acompañar el informe presentado al término del examen y concediendo que pueda expresarse una opinión basada en la veracidad de los documentos y de los estados financieros y en que no se impongan restricciones al auditor en su trabajo de investigación.

De igual forma (Gumter y Liuttobcit), exponen que:

La auditoría interna se define como el examen crítico, sistemático y detallado de un sistema de información de una unidad económica, realizado por un profesional con vínculos laborales con la organización, utilizando técnicas determinadas y con el objeto de emitir un informe y formular sugerencias para el mejoramiento de la misma. (p.33)

Lo referido permite entender que, este tipo de informes forenses son estrictamente de circulación interna y no tienen trascendencia a los terceros pues no se producen bajo la figura de la Fe Pública. Asi mismo, es importante resaltar que este tipo de clasificación también abarca otras tantas, que pueden identificarse con una o con otra, dependiendo de los vínculos que existan entre el o los auditores y la organización, entre ellas se mencionan: la Auditoría Administrativa; Auditoría Operacional; Auditoría Financiera; Auditoría Fiscal; Auditoría de Cumplimiento; Auditoría de Gestión y Resultados; Auditoría Gubernamental, entre muchas otras más específicas; todas ellas, con el mismo propósito identificar la veracidad de los procesos contables existentes evitando la posibilidad de la existencia del delito o fraude.

Lo antes descrito lleva a establecer la importancia de lo referido por Rodríguez (2016), quien establece que: 
La Auditoría Forense es aplicable dentro del contexto de un conflicto real o de una acción legal con una pérdida financiera significativa, donde el auditor forense ofrece sus servicios basado en la aplicación del conocimiento relacionado con los dominios de lo contable (información financiera, contabilidad, finanzas, auditoría y control) y del conocimiento relacionado con la investigación financiera, cuantificación de pérdidas y ciertos aspectos de ley.(p.34).

En tal sentido, se entiende que la Auditoria Forense no sólo se conforma con investigar delitos como: enriquecimiento ilícito, peculado, cohecho, soborno, malversación de fondos, etc., sino que también ha diversificado su portafolio de servicios para participar en investigaciones relacionadas con los crímenes fiscales, los crímenes contables, el crimen corporativo, el lavado de dinero y el terrorismo, entre otros.

Se hace entonces evidente, que en una sociedad desarrollada o en vías de serlo, es necesaria la implementación de técnicas manejadas por la auditoría forense, las cuales estén dirigidas a la correcta visualización de los delitos económicos, financieros y contables, así como lograr el adecuado manejo de la investigación de los mismos, lo que permitirá simultáneamente la obtención de las pruebas debidas para la segura captura de quienes los cometan.

Partiendo de dicho concepto, se brinda a la auditoría forense el respectivo valor que esta ciencia merece, considerando que en la actualidad es evidente la necesidad de implementar procesos de investigación científica y penal que le permitan a los especialistas en esta área alcanzar mayores resultados, los cuales logren minimicen la impunidad, especialmente en estos momentos tan difíciles, en los cuales el crimen organizado utiliza medios más sofisticados para lavar dinero, financiar operaciones ilícitas y ocultar los resultados de diversos delitos, considerándose que, en los tribunales penales de algunos países de América del Sur, Norteamérica y Europa, este tipo de delincuentes no son juzgados.

En razón de estos planteamientos, se establece la importancia comprendida en el presente artículo, el cual tiene como norte analizar la auditoría forense aplicada a la investigación penal. 


\section{Método.}

Para el desarrollo de este proceso investigativo, se plantea como metodología encaminada hacia una orientación científica particular que se encuentra determinada por la necesidad de indagar en forma precisa y coherente una situación, en tal sentido Dávila (2015) define la metodología “como aquellos pasos previos que son seleccionados por el investigador para lograr resultados favorables que le ayuden a plantear nuevas ideas". (p.66)

Lo citado por el autor, lleva a entender que el desarrollo de la acción investigativa busca simplemente coordinar acciones enmarcadas en una revisión bibliográfica con el fin de complementar ideas previas relacionadas con la auditoría forense aplicada a la investigación penal, para así finalmente elaborar un cuerpo de consideraciones generales que ayuden a ampliar el interés propuesto.

\section{Tipo de Investigación}

Dentro de toda práctica investigativa, se precisan acciones de carácter metodológico mediante las cuales, se logra conocer y proyectar los eventos posibles que la determinan, así como las características que hacen del acto científico un proceso interactivo ajustado a una realidad posible de ser interpretada. En este sentido, se puede decir, que la presente investigación corresponde al tipo documental, definido por Castro (2016), "se ocupa del estudio de problemas planteados a nivel teórico, la información requerida para abordarlos se encuentra básicamente en materiales impresos, audiovisuales y /o electrónicos”. (p.41).

En consideración a esta definición, la orientación metodológica permitió la oportunidad de cumplir con una serie de actividades inherentes a la revisión y lectura de diversos documentos donde se encontraron ideas explicitas relacionadas con los tópicos encargados de identificar a cada característica insertada en el estudio. Por lo tanto, se realizaron continuas interpretaciones con el claro propósito de revisar aquellas apreciaciones o investigaciones propuestas por diferentes investigadores relacionadas con el tema de interés, para luego dar la respectiva argumentación a los planteamientos, en función a las necesidades encontradas en la indagación. 


\section{Fuentes Documentales}

El análisis correspondiente a las características que predomina en el tema seleccionado, llevan a incluir diferentes fuentes documentales encargadas de darle el respectivo apoyo y en ese sentido cumplir con la valoración de los hechos a fin de generar nuevos criterios que sirven de referencia a otros procesos investigativos. Para Castro (ob.cit) las fuentes documentales incorporadas en la investigación documental o bibliográfica, "representa la suma de materiales sistemáticos que son revisados en forma rigurosa y profunda para llegar a un análisis del fenómeno”.(p.41). Por lo tanto, se procedió a cumplir con la realización de una lectura previa determinada por encontrar aquellos aspectos estrechamente vinculados con el tema, para luego explicar mediante un desarrollo las respectivas apreciaciones generales de importancia.

\section{Técnicas para la Recolección de la Información}

La conducción de la investigación para ser realizada en función a las particularidades que determinan a los estudios documentales, tiene como fin el desarrollo de un conjunto de acciones encargadas de llevar a la selección de técnicas estrechamente vinculadas con las características del estudio. En tal sentido, Bolívar (2015), refiere, que es "una técnica particular para aportar ayuda a los procedimientos de selección de las ideas primarias y secundarias”. (p. 71).

Por ello, se procedió a la utilización del subrayado, resúmenes, fichaje, como parte básica para la revisión y selección de los documentos que presentan el contenido teórico. Es decir, que mediante su aplicación de estas técnicas se pudo llegar a recoger informaciones en cuanto a la revisión bibliográfica de los diversos elementos encargados de orientar el proceso de investigación. Tal como lo expresa, Bolívar (ob.cit) "las técnicas documentales proporcionan las herramientas esenciales y determinantes para responder a los objetivos formulados y llegar a resultados efectivos" (p. 58). Es decir, para responder con eficiencia a las necesidades investigativas, se introdujeron como técnica de recolección el método inductivo, que hizo posible llevar a cabo una valoración de los hechos de forma particular para llegar a la explicación desde una visión general.

Así mismo, se emplearon las técnicas de análisis de información para la realización de la investigación que fue ejecutada bajo la dinámica de aplicar diversos elementos encargados de determinar el camino a recorrer por el estudio, según, Bolívar (ob.cit) las técnicas de procesamiento

\footnotetext{
703 Vol. 5, núm. 1, enero 2019, pp. 695-712

Dewis E. Álvarez-Pincay; Sandra P. Tóala-Bozada; Zoila M. Delgado-Gutierrez; José F. Peñafiel-

Loor; Adela del Jesús Lucio-Pillasagua; Mercy K. Moreira-Cañarte
} 
de datos en los estudios documentales "son las encargadas de ofrecer al investigador la visión o pasos que debe cumplir durante su ejercicio, cada una de ellas debe estar en correspondencia con el nivel a emplear" (p. 123). Esto indica, que para llevar a cabo el procesamiento de los datos obtenidos una vez aplicado las técnicas seleccionadas, tales como: fichas de resumen, textual, registros descriptivos entre otros, los mismos se deben ajustar al nivel que ha sido seleccionado.

\section{Resultados.}

\section{Auditoría Forense}

La Auditoría Forense no puede apreciarse como una técnica nueva ni constituye el nacimiento de una nueva ciencia, es la conjunción de varias ciencias encabezadas por la Contaduría Pública y el Derecho. De allí, que para poder definir lo que es la Auditoría Forense, se hace necesario en principio conocer el significado de ambos términos por separado. Para Becerra (2015), "la Auditoría es una de las aplicaciones de los principios científicos de la contabilidad, se basa en la verificación de los registros patrimoniales de las haciendas y organizaciones, para observar su exactitud". (p.34).

Entiéndase a través de este señalamiento que, la auditoría forense representa una actividad por la cual se verifica la corrección contable de las cifras de los estados financieros, es decir, se basa en la revisión misma de los registros y fuentes de contabilidad, para determinar la racionabilidad de las cifras que muestran los estados financieros emanados de ellos. Esta ciencia, comprende un estudio especializado de los documentos que soportan legal, técnica, financiera y contablemente las operaciones realizadas por los responsables de la información financiera en la empresa, durante un período determinado.

Dicho de otra forma, la auditoría forense representa una función independiente de la evaluación establecida dentro de una organización, para examinar y evaluar sus actividades como un servicio a cada conjunto organizacional. La misma, amerita del estudio y evaluación del control interno, el cual se basa en la protección a través de todos los instrumentos pertinentes, de la cobertura adecuada de las posibles contingencias y de la verificación de los sistemas de preservación y registro.

En apoyo a lo expuesto, Lerutt (2014), expresa: 
La auditoría forense es una disciplina especializada que requiere un conocimiento experto en teorías contables, de auditoría y técnicas de investigación criminal. Es una rama importante de contabilidad investigativa, utilizada en la reconstrucción de hechos financieros, investigaciones de fraudes, cálculos de daños económicos y rendimientos de proyecciones financieras. (p.95)

Lo descrito lleva a comprender que, la auditoría forense es una ciencia especializada en descubrir, divulgar y atestar sobre fraudes y delitos en el desarrollo de las funciones públicas y privadas. También, estudia los efectos de hechos que pueden ser delictivos o no, para aportar al juzgador las pruebas en donde se involucran registros de contabilidad, pruebas eminentemente técnicocientíficas, y análisis jurídico-contables que facilitan el trabajo de la administración de justicia.

Del mismo modo, Lerutt, (obcit), establece:

El auditor forense va más allá de la evidencia de auditoría, de la seguridad razonable, evalúa e investiga al ciento por ciento, centrándose en el hecho ilícito y en la mala fe de las personas, involucra por lo menos: análisis, cuantificación de pérdidas, investigaciones, recolección de evidencia, mediación, arbitramento y testimonio como un testigo experto. $(\mathrm{p}, 96)$

En otras palabras, representa una ciencia logística y profesional que permite reunir y presentar información financiera, contable, legal, administrativa e impositiva, en una forma que será aceptada por una corte de jurisprudencia contra los perpetradores de un crimen económico, es decir, constituye una rama importante de la contabilidad investigativa utilizada en la reconstrucción de hechos financieros, investigaciones de fraudes, cálculos de daños económicos y rendimientos de proyecciones financieras. Por ende, esta ciencia es concebida con un carácter plenamente penal, por cuanto se fundamenta en varias razones como lo son:

a) su génesis es de orden procesal penal

b) está considerada en el marco de las disciplinas auxiliares penales

c) su aplicación científica contribuye para conocer los hechos y llegar a las penalidades determinadas por los jueces. 
A la vez es auxiliar de la Criminalística, puesto que no determina responsabilidades, ni señala directamente penalidades, sino que realiza investigaciones para conocer los hechos y presentar pruebas respecto a su ejecución, desarrollo y consumación. En pocas palabras, se trata de un procedimiento técnico-científico que permite que un experto emita ante los jueces conceptos y opiniones de valor técnico, especialmente en lo relativo a la vigilancia de la gestión fiscal, financiera y monetaria de una organización bien sea pública o privada; de esta manera, se contribuye a mejorar las economías de los países.

En el orden de lo establecido, es importante resaltar que la auditoría forense comprende dos tipos de enfoques que coadyuvan a determinar cuál será el plan de acción en una organización para el tratamiento del problema de fraude, los mismos definen de la siguiente forma:

- Auditoría Forense Preventiva. Orientada a proporcionar aseguramiento (evaluación) o asesoría a las organizaciones respecto a su capacidad para disuadir, prevenir (evitar), detectar y reaccionar ante fraudes financieros, puede incluir trabajos de consultoría para implementar: programas y controles antifraude, esquemas de alerta temprana de irregularidades y sistemas de administración de denuncias. Este enfoque es proactivo por cuanto implica tomar decisiones y acciones en el presente, para evitar fraudes en el futuro.

- Auditoría Forense Detectiva. Orientada a identificar la existencia de fraudes financieros mediante la investigación profunda de los mismos, establece, entre otros aspectos: cuantía del fraude; efectos directos e indirectos; presuntos autores, cómplices y encubridores; en muchas ocasiones los resultados de un trabajo de auditoría forense detectiva son puestos a consideración de la justicia que se encargará de analizar, juzgar y dictar la sentencia respectiva. Este enfoque es reactivo por cuanto implica tomar acciones y decisiones en el presente respecto a fraudes sucedidos en el pasado.

En tal sentido, se debe entender que el Auditor Forense no es un simple tenedor de libros o un auditor rutinario, este profesional requiere de las mismas destrezas básicas que se necesitan para ser un buen auditor, más habilidades especiales como para prestar atención a los detalles mínimos, analizar información en forma exhaustiva y dominar elementos básicos del procesamiento de datos. En pocas palabras, el auditor forense analiza, interpreta, recopila y presenta complejas finanzas y los 
flujos de manera que sean entendibles, como correctamente sustentados con el fin de asistir a la justicia en los aspectos contables.

\section{La Auditoría Forense aplicada a la Investigación Penal}

El conocimiento total de una empresa, y el manejo de su control interno, financiero y fiscal o tributario, permite dejar al descubierto los posibles focos donde se pueda presumir la comisión de los delitos que se persiguen; es decir, que permitan hacer referencia a los síntomas o establecer indicadores de fraudes organización. Para ello, es recomendable seguir las siguientes fases:

\section{Fase I. Planificación}

Esta fase incluye el conocimiento de la empresa, tanto en su entorno externo como interno. Permite delimitar el trabajo a realizar, estableciendo de manera concreta los objetivos que se pretenden alcanzar, así como el plan de trabajo a seguir. Facilita el delimitar el período a investigar, las áreas, el personal y las fuentes que formarán parte de la investigación.

A través del desarrollo de esta Fase, se facilita la elaboración del plan de trabajo, precisando los programas a emplear, técnicas necesarias y finalmente definiendo los objetivos que se pretenden alcanzar, considerando además las directrices establecidas por el contratante. Por ello, para garantizar los resultados propuestos se recomienda seguir las pautas que se señalan a continuación:

- Evaluación de la Auditoría previa. Iniciada la fase del diseño, es necesario realizar un estudio pormenorizado de la Auditoría Financiera realizada en la etapa preliminar, de manera que se puedan destacar los posibles fraudes percibidos en esta etapa y los puntos débiles de la empresa, pudiendo con esto realizar el análisis y reconocimiento del problema que pasa a ser objeto de la Auditoría Forense.

- Definición y reconocimiento del problema. Consiste en investigar los indicios observados en la Auditoría Financiera, que muestren la posibilidad de la existencia de la comisión del hecho delictivo. Al verificar estos detalles e identificar de manera clara y plena a la empresa $u$ organización, así como a sus directores o accionistas, cuya participación y reacción a la investigación permitirá avanzar en la misma 
- Elaboración del plan de trabajo. Una vez determinado el problema y definida la organización, se podrá entonces diseñar el plan de trabajo a seguir, con determinación del tiempo a emplear, las partidas a investigar, la cantidad de personal necesario, así como el diseño de los formatos requeridos para la obtención de las evidencias y pruebas.

\section{Fase II. Ejecución}

Realización del plan de trabajo. Esta fase abarca todo lo relacionado con el trabajo de campo y consiste en llevar a la práctica los planes elaborados en la fase anterior. Se inicia con la preparación de los papeles de trabajo, los cuales son prueba fundamental al momento de emitir una opinión. En ellos se reflejan de manera detallada el trabajo de los auditores y las evidencias obtenidas en la investigación; es por esta razón, que dichos papeles deben ser muy claros y específicos en cuanto al punto que se desarrolla. Una vez diseñados o definidos los formatos a utilizar y puesto en marcha el plan de trabajo, es necesario verificar con detenimiento el cumplimiento del control interno y de los riesgos que crean en la organización puntos vulnerables para delinquir.

Recopilación de evidencias de fraude. Se corresponde con la búsqueda de evidencias suficientes para garantizar el éxito de la investigación, recopilando las evidencias necesarias para poder determinar si el fraude ha tenido lugar o no. Esta normativa no ha sido elaborada de manera específica para la Auditoría Forense, sin embargo, su fundamento sirve igualmente de base, pues al complementarlo con la regulación y los principios del derecho probatorio, es posible obtener evidencias convertibles en pruebas. Dichas evidencias pueden y deben ser complementadas con herramientas tecnológicas como:

a) fotografías (tomadas con las técnicas forenses)

b) huellas dactilares, pruebas de caligrafía

c) video grabaciones con cámara oculta selladas, con programación de tiempo y fecha

d) audio-grabación y verificación de voz

e) rastreo de información en la memoria y archivos de los computadores

f) (d) recuperación de datos borrados del disco duros en los computadores, entre muchos otros.

Evaluación de la evidencia recolectada. Las evidencias son recogidas para determinar Quién, Qué, Cuánto, Dónde, Cómo, Por qué y Cuándo se ha cometido el fraude. De manera que, al momento de 
evaluarlas, se debe observar si además de ser pertinentes, suficientes y competentes, responden estas incógnitas y cumplen con los criterios de admisibilidad jurídica, necesaria para ser convertidas en Pruebas. Es decir, deben ser de tipo circunstancial, testimonial o documental, poseer soporte físico y haber sido obtenidas con técnicas periciales, de manera consciente, libre de coacción, violencia, dolo, cohecho o seducción.

\section{Fase III. Informe Pericial o Experticia}

La redacción del Informe Forense debe realizarse de acuerdo con lo estipulado en las Normas de Auditoría Generalmente Aceptadas y en las Normas Internacionales de Auditoría. Por ende, deben contener:

a) los antecedentes de la investigación

b) la descripción de los procedimientos y pruebas practicadas

c) un análisis de las evidencias o pruebas obtenidas

d) un capítulo en donde se establecen las conclusiones y las recomendaciones específicas del caso investigado.

Este informe es la principal evidencia de valor legal o prueba que se puede presentar ante un tribunal, pues de su claridad y correcta elaboración dependerán las resultas en los juzgados. Es por ello que en su elaboración se debe tener en cuenta que debe ser preciso, oportuno, exhaustivo, imparcial, claro, relevante y completo.

Para que el informe forense exprese de manera correcta las conclusiones necesarias, las mismas deben:

1. Especificar la evidencia recopilada de los hechos pertinentes y las presunciones usadas.

2. Explicar los factores importantes considerados para formular las conclusiones.

3. Incluir las labores pertinentes, los horarios, el informe del documento y otra información útil.

4. Comunicar los resultados del trabajo por parte del auditor forense y/o la opinión de los problemas sometidos.

Además, en la estructura del informe debe presentarse información referente al estado en el que se encuentra la organización a la fecha del informe; la identificación del auditor forense y de la

\footnotetext{
709 Vol. 5, núm. 1, enero 2019, pp. 695-712 
organización y sobre todo las restricciones en el uso del informe y las limitaciones de la conclusión. La naturaleza, volumen y forma del informe pueden variar, dependiendo de la naturaleza del trabajo y del propósito objetivo e intencional del informe.

En virtud de los planteamientos dados se establece que, la Auditoría Forense no debe ser considerada como una rama de la Contaduría Pública, sino como una nueva ciencia nacida de la conjunción de las ciencias contables y las ciencias jurídicas, a través de la cual, se obtiene una herramienta óptima y sistemática, cuyo resultado podría ser utilizado en un litigio como fundamento de la sentencia.

\section{Conclusiones.}

El estudio de la Auditoría Forense, constituye un tema novedoso e interesante desde distintos puntos de vista. El primero referido a la sociedad, porque tendría la oportunidad de demostrar que han sido violados los derechos de sus integrantes, con la comisión de fraudes y delitos, logrando a su vez el castigo para los perpetradores de los mismos. Desde el punto de vista del orden jurídico, es importante pues con su existencia se logra la homogenización del sistema jurídico, en materia de prueba para los delitos que fueron objetos de estudio, su ampliación en la presentación de pruebas y el realizado por los expertos contables, adscritos a los cuerpos y organizaciones adscritas a las Investigaciones Científicas, Penales y Criminalísticas correspondientes, De igual manera, es una herramienta interesante para todas las organizaciones, ya que su existencia y posible aplicación garantizará la defensa de sus derechos y de su patrimonio, durante un juicio.

Desde este ángulo, se puede concluir que para que la auditoria forense logre contribuir al castigo y por ende reducción de delitos como el lavado de activos, la financiación del terrorismo, el fraude financiero, la estafa, el testaferrato, el detrimento del erario público, es necesario que las facultades de contaduría pública incluyan este tema en sus programas educativos, que se creen espacios de estudios de investigación en esta rama y además que haya un ente regulador del mismo, que se creen estándares con el fin de darle la relevancia que amerita esta especialidad, que se dé el reconocimiento a la profesión en esta especialidad y el alcance de la misma, que se controle la calidad profesional de los contadores de una manera más efectiva y finalmente, que exista conciencia profesional sobre la relevancia de la profesión y su utilidad dentro de la sociedad.

\footnotetext{
\begin{tabular}{l|l}
710 & Vol. 5, núm. 1, enero 2019, pp. 695-712 \\
Dewis E. Álvarez-Pincay; Sandra P. Tóala-Bozada; Zoila M. Delgado-Gutierrez; José F. Peñafiel-
\end{tabular} Loor; Adela del Jesús Lucio-Pillasagua; Mercy K. Moreira-Cañarte
} 
Por consiguiente, es necesario considerar que la Auditoria Forense se presenta como un nuevo rol para el contador público, donde una vez más se da la oportunidad al profesional de seguir ampliando sus conocimientos y adquiriendo nuevas experiencias, al mismo tiempo para aquellos estudiantes de contaduría pública que quieran profundizar, indagar y terminar siendo un experto para colaborar a la justicia colombiana.

Por tal razón, para ser un buen auditor forense se necesita ser un buen contador público y para poder ejercer la Auditoria Forense se requiere del conocimiento legal, la integración de la contabilidad, la auditoria y la habilidad de investigación, por esto el auditor forense evalúa el desarrollo de técnicas forenses como la documentología, dactiloscopia, grafología y aplicaciones sistematizadas consideradas como elementos de prueba ante la corte. Pues en la Auditoria Forense lo importante de la prueba es que conduzca a la verdad; de allí, que si un investigador no tiene profundos conocimientos en los temas jurídicos, contables y financieros, difícilmente aportará las pruebas contundentes, que requiere la justicia para llegar a sentencias condenatorias.

\section{Bibliografía.}

Barrett, M. (2015). El contador público como investigador forense. Ponencia presentada en el X Congreso Venezolano de Contaduría Pública, Venezuela.

Becerra, H. (2015). Teoría general y Paradigmas Vanguardistas de la prueba judicial. Buenos Aires: Zavalía.

Bolívar, E. (2015). Metodología de la Investigación, Métodos y Enfoques. Caracas editorial: Panapo

Castro, E. (2016). Enfoque y Diseño de la Investigación Científica, Tomo II. Barcelona, España: Bosh.

Dávila, J. (2015). Principios y Enfoques de la Investigación (Tomo II). Madrid: Editorial Las Garzas

Gumter, M. y Liutt, R. (2014). Tipos de Auditores. Revista Betsime del Empresario Cubano [Revista en línea]. Disponible: http://www.betsime.disaic.cu/secciones/

Kartt, R. (2015). Auditoría forense [Documento en línea]. Federación Latinoamericana de Auditores Internos. Disponible: http://www.laflai.org

Lerutt, D. (2004). Propuesta de una metodología en auditoría forense para el delito desviación presupuestaria. Tesis de grado de especialización, Universidad de San Martín de Porres ubicada en Lima Perú 
Rodríguez, J. (2016). Métodos de Investigación de la Auditoría Forense. España: Océano Centrum.

Venziatt, C. (2014). El Fraude como elemento decisivo en la Auditoría Forense. Artículo de Economía de la Facultad de Economía de la Universidad Nuevo México en México 